- The themes from qualitative analysis included the benefit of common language and sense of urgency that came across from describing the changes in PPS and POI over a period of time.

Conclusions Palliative Care Performance Status and Phase of Illness can be implemented across an acute hospital and can aid transitions to discharges. It improves communication of the patients needs, health and carer status. As communication underpins all we do the need for accurate, reproducible, evidence based descriptors is important to keep patients safe and to advocate for their needs.

\section{IMPROVEMENT OF MDT MEETINGS IN A PALLIATIVE CARE INPATIENT SETTING - A SERVICE IMPROVEMENT PROJECT}

Sarah Shipman, Constantina Pitsillides. Sheffield Teaching Hospitals

10.1136/bmjspcare-2019-ASP.150

Background Multidisciplinary team (MDT) meetings are central to the management of chronic disease and they have become widely established across the NHS (Department of Health, 2004). MDT meetings are believed to ensure higher quality decision-making and improved outcomes however the evidence to support this is limited.

Despite MDT care being found in many secondary care settings throughout the UK, there is no national standard for an MDT (NICE, 2017). NICE suggests that indeed some of the MDT's success may be in the flexibility to suit each particular clinical area; however, good planning and communication are common themes throughout.

The Macmillan Palliative Care Unit (MPCU) holds a weekly MDT to discuss all current inpatients. There is opportunity to review the effectiveness of documentation during these meetings to ensure they are fit for purpose.

Methods Data was collected retrospectively looking at various aspects of good record keeping prior to a MDT proforma being implemented. A proforma was then introduced and changes made each week on 3 separate weeks. Data was collected after each change to monitor if record keeping improved. Changes made were based on suggestions by members of the MDT.

Results Number of patients' notes reviewed ranged from 1418 depending on the number of patients on the inpatient unit that week. A deterioration or poor documentation in specific areas prompted changes to be made to the proforma. As a result, the overall documentation of discussions and decisions greatly improved.

Conclusion Overall documentation of discussions and decisions made at MDT meetings has improved following the implementation of the proforma and ensures all MDT members are included in discussions about patients.

\section{TRANSFORMING INTERPROFESSIONAL WORKING IN AN ACUTE PALLIATIVE CARE SERVICE}

Kim Steel, Monica Keenan, Marianne MacLeod, Fran Stretton. NHS Fife

10.1136/bmjspcare-2019-ASP.151

Background Since 2016 NHS Fife acute palliative care team has been developing models of interprofessional working within the acute hospital. The NHS Fife Specialist Palliative Care Service is a single NHS service which includes 2 hospice units, a community and acute team. Although the Specialist Palliative Care service provides medical and nursing input into the acute hospital, the hospital provides occupational therapy, dietetic, chaplaincy and pharmacy support. To ensure we were providing effective and efficient service we have undertaken multiple assurance measures about the service

Methods

1. Footprint of mortality across our hospital-this was to inform which areas we needed to focus opportunities to develop to benefit most patients;

2. Setting up a structured acute palliative care MDT with all groups that obligates commitment to the service defines the activities of the professionals that attend the group. The MDT also includes learning from complaints, comments and compliments;

3. Using Palliative Performance Score (PPS) and Phase of Illness (POI) to describe our patient group to provide re-assurance that we are seeing patients of appropriate complexity;

4. Developing governance reporting mechanisms from our MDT activity.

Results By understanding where the highest mortality is within our hospital we have been able to increase the focus of resource and education into that area. The MDT has resulted in increase in the confidence of the professionals to overcome organisational boundaries results in co-ordination and efficiency of our approach to patients. PPS and POI has shown that we see mostly unstable patients across the range of PPS and POI. We have annual audit activity that reports to the acute operating divisions governance structure.

Conclusions Sharing a structured MDT and using a system for discussing patients has resulted in sufficient improvements in co-ordination of our acute palliative care team. The change in interprofessional monitoring and reporting has raised the profile and impact of acute palliative care.

\section{Supportive Care | Posters 129 - 139}

\section{EXPLORATION OF VOLUNTEERS AND SUPPORT WORKERS INITIATION OF QUALITY OF LIFE CONVERSATIONS IN HOSPICE PALLIATIVE DAY CARE}

Sharan Watson, Alison Hembrow. University of Derby, Treetops Hospice Care

10.1136/bmjspcare-2019-ASP.152

Background Treetops Hospice, in partnership with University of Derby have commenced a research project exploring the outcomes of developing volunteers, in initiating conversations around quality of life. As a pilot site work for NHS England for Personal Health Budgets (PHB's), Treetops discovered that the 'conversations' around what's important right now to the patient/carer can be just as important, than the outcome of the PHB. This is a vital piece of work which directly could impact on improving individual's wellbeing, the new approach focuses around the mnemonic L.I.S.T.E.N (developed by Treetops Hospice). Policy drivers have acknowledged that there is a much greater need and demand for person centred care than professionals in health and social care can meet, the barriers around developing these conversations, possibly relate to lack of time and clarity to 
whose role it is. Volunteers and support workers may be advantageous in having these conversations with the right support and development.

Methods There is a mixed methods approach to this research in two separate stages, involving 14 volunteers and support staff.

First stage Face to face qualitative interviews prior to exposure of new training/model of developing these conversations and pre-training Likert questionnaire have been undertaken.

Results Thematic analysis using NVIVO, to address study objectives from first stage, has identified variables in how participants engage with individuals prior to this support and training. Emerging themes are: discussions around whose role is it; feeling valued within their role; being given 'permission' and motivation around having these conversations.

Conclusions Second stage is now being undertaken, emerging themes will be presented in APM Conference. This stage will focus on identifying whether a different approach has helped, and volunteers' feedback will inform training developments and dissemination about how to support volunteers in having honest conversations about quality of life.

\section{FRAILTY SCORING IN PATIENTS WITH END STAGE RENAL FAILURE}

L Hetherington, J Prentice, M Findlay, T Collidge. NHS Greater Glasgow and Clyde, Scottish Renal Palliative Collaberative Group, The Glasgow Renal and Transplant Unit, South Glasgow University Hospital, The Beatson West of Scotland Cancer Centre, The University of Glasgow

\subsection{6/bmjspcare-2019-ASP.153}

Background In the end stage renal failure (ESRF) population frailty is associated with early mortality, increased hospitalisations, and significant symptom burden. After identifying a high degree of frailty in patients withdrawing from dialysis in our population we examined the use of formal frailty scoring and its use in identifying deteriorating patients on renal replacement therapy (RRT).

Methods The Rockwood Clinical Frailty Scale (CFS) is a 9point scale which enables measurement of frailty it has high inter-rater reliability and correlates well with objective measures of frailty and has been validated in the ESRF population. We introduced routine recording of the CFS for all ESRF in our region for patients at three monthly intervals and for low clearance patients at the time of RRT education.

Results A total of 1663 scores (range 1-9) have been recorded in 798 patients. Mean age 63.9 years. Of those patients currently undergoing haemodialysis the median CFS score was 4 $(n=533)$. The median score prior to death was 5.5. Evidence of deterioration in CFS score (last score greater than the first) was present in $50 \%(14 / 28)$ of those who were deceased at follow-up, whereas a deteriorating score was only present in $22.3 \%(97 / 435)$ of those who remained alive, $p=0.001$. A documented score of 6 or greater was present in $51.4 \%$ (38/ 74 ) of those who subsequently died vs. $21.7 \%(158 / 727)$ of those who remained alive, $\mathrm{p}<0.001$.

Conclusion Deterioration in frailty score is associated with death at follow-up. Furthermore, a score 6 or greater at any point is predictive of death at follow-up. Routine monitoring of frailty using the CFS provides a simple tool to identify patients who are deteriorating and at risk of death. High or deteriorating CFS score should trigger clinical review and anticipatory care planning where appropriate.

\section{A PROSPECTIVE COHORT STUDY DESCRIBING THE STABILITY OF CARE PREFERENCES IN FRAIL OLDER PEOPLE FOLLOWING ACUTE ILLNESS}

Simon Noah Etkind, Anna E Bone, Fliss EM Murtagh, Irene J Higginson. King's College London, Cicely Saunders Institute; Wolfson Palliative Care Research Institute, Hull York Medical School

\subsection{6/bmjspcare-2019-ASP.154}

Background Care should be responsive to preferences but there is little evidence regarding the stability of care preferences, particularly in frail older people after acute illness.

Aim To describe the stability of preferred care outcomes in frail older people in the six months following acute illness.

Methods Prospective cohort study of frail older adults with recent acute illness requiring secondary care review or admission, surveyed at three time-points over six months. Participants rated the importance of six preferred care outcomes chosen from literature review (to extend life, be comfortable, improve quality of life, remain independent, support others, stay out of hospital) on a 0-4 Likert scale, and were then asked to prioritise the most important. We used descriptive statistics to analyse preferences at baseline and the stability of preferences during the study.

Results 67 participants; 63\% female, mean age 84 (standard deviation 7.41). 10 participants (15\%) died during the study. At baseline, all preferred outcomes were rated mean $>3.3 / 4$ in importance, except 'to extend life' (2.08/ 4). Most frequent baseline priorities were: stay out of hospital (20\%), support others (16\%), and improve quality of life $(16 \%)$. During the study, the importance ascribed to each preferred outcome was largely stable, but what was most important changed at $66 \%$ of $n=85$ opportunities. Overall, being comfortable became most important for more patients during the study (increasing from 5\% to 14\%, (Chi2 4.41, p=0.036)), whilst there was a non-significant trend away from staying out of hospital being most important (27\% to $22 \%)$.

Conclusions For frail older people, the importance of preferred care outcomes remains stable after acute illness, but the outcome ranked as most important frequently changes. Due to its stability, the level of importance ascribed to preferred outcomes may be more useful in clinical practice and advance care planning.

\section{DESCRIBING DEPRESCRIBING - WHEN ARE WE STOPPING MEDICATIONS IN PALLIATIVE CARE?}

Matthew Doré, Hannah Fox, Trish Campbell, Derek Willis. Severn Hospice

10.1136/bmjspcare-2019-ASP.155

Introduction Deprescribing is the process of withdrawal of medication with the goal of improving patient care. Research currently is in the context of polypharmacy and geriatrics, however despite its ubiquity within palliative care there is a paucity of research in this context. Anecdotally, deprescribing is performed adhoc and using 'common sense' but there appears no clear data on rational, benefit and whole numbers involved.

Aim To gather data on current deprescribing practice within palliative care, with the aim to inform our own specialty and others. 